\title{
Free modal algebras: a coalgebraic perspective
}

\author{
N. Bezhanishvili ${ }^{1 \star} \quad$ A. Kurz ${ }^{1 \star \star}$ \\ Department of Computer Science, University of Leicester, United Kingdom
}

\begin{abstract}
In this paper we discuss a uniform method for constructing free modal and distributive modal algebras. This method draws on works by (Abramsky 2005) and (Ghilardi 1995). We revisit the theory of normal forms for modal logic and derive a normal form representation for positive modal logic. We also show that every finitely generated free modal and distributive modal algebra axiomatised by equations of rank 1 is a reduct of a temporal algebra.
\end{abstract}

\section{Introduction}

Modal logics play an important role in many areas of computer science. In recent years, the connection of modal logic and coalgebra received a lot of attention, see eg [30]. In particular, it has been recognised that modal logic is to coalgebras what equational logic is to algebras. The precise relationship between the logics and the coalgebras can be formulated using Stone duality [9]. From this perspective, algebras are the logical forms of coalgebras [1]; and the algebras that appear in this way give rise to modal logics.

In this paper we take the opposite view and ask how coalgebraic and categorical methods can elucidate traditional topics in modal logic. Algebraic methods and techniques proved to be very useful in investigations of modal logics, see eg [8,30]. Here we apply a mix of algebraic and coalgebraic (and categorical) techniques to shed some light on the construction of canonical models of modal logics. In principle, almost all properties of a given modal logic are enshrined in its free modal algebras or, dually and equivalently, in its canonical models [8]. Therefore, an understanding of the structure of the canonical model of a given modal logic can be the key for understanding the properties of this logic.

The general idea that we will discuss in this paper has appeared before in different contexts. Fine [16] used his canonical formulas for describing canonical models of modal logics and for deriving completeness results for these logics. Moss [25] revisited Fine's formulas to give a filtration type finite-model property proofs for various modal logics. Abramsky [2] constructed the canonical model of closed formulas of the basic modal logic as the final coalgebra for the Vietoris functor and Ghilardi $[18,17]$ gave a similar description of canonical models of modal and intuitionistic logics to derive a normal form representation for these logics. For positive modal logic similar techniques were developed by Davey and Goldberg [13].

\footnotetext{
* Supported by the EPSRC grant EP/C014014/1 and by the Georgian National Science Foundation grant GNSF/ST06/3-003.

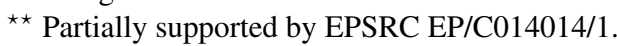


The aim of this paper is to unify all these approaches and present a coherent method for constructing free modal and distributive modal algebras. Modal algebras are algebraic models of (classical) modal logic and distributive modal algebras are algebraic models of positive (negation-free) modal logic. We will show how to construct free algebras for a variety $\mathbf{V}$ equipped with an operator $f$. In case of modal algebras $\mathbf{V}$ is the variety of Boolean algebras and in case of distributive modal algebras $\mathbf{V}$ is the variety of distributive lattices. The main idea of the construction is the following: We start with the free $\mathbf{V}$-algebra and step by step add freely to it the operator $f$. As a result we obtain a countable sequence of algebras whose direct limit is the desired free algebra.

We apply this general method to modal and distributive modal algebras. For distributive modal algebras these results appear to be new. In case of modal algebras this approach gives simple and coherent proofs of known results. We use the Stone duality for Boolean algebras and the Priestley duality for distributive lattices to describe the dual spaces of the finite approximants of the free algebras. The key for dualising these constructions lies in the coalgebraic representation of modal spaces as coalgebras for the Vietoris functor [22] and in the coalgebraic representation of modal Priestley spaces as coalgebras for the convex set functor [20,27]. This allows us to represent the canonical models of modal and positive modal logic as a limit of finite sets and posets, respectively. We also observe that the underlying Stone space of the canonical model of the basic modal logic is homeomorphic to the so-called Pelczynski space. This space appears to be one of the nine fixed points of the Vietoris functor on compact Hausdorff spaces with a countable basis [28, 26].

As we will see below, this method directly applies to modal and positive modal logics that are axiomatised by the formulas of rank 1 . We also indicate how to adjust our techniques to modal logics that are not axiomatised by formulas of rank 1. As an example we consider the 'reflexive' modal logic, that is, the modal logic axiomatised by the additional reflexivity axiom $\varphi \rightarrow \diamond \varphi$, which is not of rank 1 . This example also highlights how the Sahlqvist correspondence-an important technique of modal logic — can be applied to our method in order to describe canonical models of modal logics that are not axiomatised by formulas of rank 1 .

In the end of the paper we revisit Fine's normal forms for modal logic in a manner similar to Abramsky [2] and Ghilardi [18] and derive normal forms for positive modal logic. We also generalise Ghilardi's result that every free modal algebra is a reduct of a temporal algebra to all varieties of modal and distributive modal algebras axiomatised by formulas of rank 1 .

Other Related Work Canonical models of modal logics have been investigated quite thoroughly. However, these investigations mostly concentrated on transitive modal logics; that is, modal logics with transitive Kripke frames. For an overview of these results we refer to [12, Section 8.6 and 8.7] (see also [7, Chapter 3] for similar results in the case of intuitionistic logic). The method of constructing canonical models for transitive modal logics is based on building the canonical model of a given logic layer by layer, that is, inductively on the depth of the canonical model. Although very useful, this method does not go through for non-transitive modal logics. For building free algebras for non-transitive modal logics one needs to use a different approach. 
Acknowledgements We would like to thank Dimitri Pataraia, David Gabelaia, Mamuka Jibladze, Leo Esakia, Mai Gehrke and Hilary Priestley for many interesting discussions. We are also very grateful to the anonymous referees for valuable suggestions.

\section{Dualities for Boolean algebras and distributive lattices}

In this section we briefly recall the Stone duality for Boolean algebras and the Priestley duality for distributive lattices.

\subsection{Stone duality for Boolean algebras}

A Stone space is a 0-dimensional (a topological space with a basis of clopens) compact Hausdorff space. For every Stone space $X$ let $\operatorname{Clp}(X)$ denote the set of clopens (closed and open subsets) of $X$. We also let $\mathcal{P}(X)$ denote the powerset of $X$. The next theorem states the celebrated Stone representation theorem.

Theorem 2.1. (eg [19, 4.4], [14, 11.4]) For every Boolean algebra B there is a Stone space $X_{B}$ such that $B$ is isomorphic to $\left(\operatorname{Clp}\left(X_{B}\right), \cup, \cap,-, \emptyset\right)$. If $B$ is finite then $X_{B}$ is finite and $\operatorname{Clp}\left(X_{B}\right)=\mathcal{P}\left(X_{B}\right)$.

Proof. (Sketch) Let $B$ be a Boolean algebra. Let $X_{B}:=$ the set of all maximal filters of $B$. For $a \in B$ let $\widehat{a}=\left\{x \in X_{B}: a \in x\right\}$. We declare $\{\widehat{a}: a \in B\}$ to be a basis for a topology on $X_{B}$. Then $X_{B}$ becomes a Stone space and $B$ is isomorphic to $\operatorname{Clp}\left(X_{B}\right)$.

Let BA denote the category of Boolean algebras and Boolean homomorphisms. Let also Stone denote the category of Stone spaces and continuous maps. The Stone representation theorem can be extended to corresponding categories.

Theorem 2.2. (see eg $[19,4.4]) \quad \mathbf{B A} \simeq$ Stone $^{o p}$.

Proof. (Sketch) By Theorem 2.1 one only needs to deal with morphisms. Let $f: X \rightarrow$ $Y$ be a continuous map. Then $f^{-1}: \mathrm{Clp}(Y) \rightarrow \mathrm{Clp}(X)$ is a Boolean homomorphism. Conversely, if $h: A \rightarrow B$ is a Boolean homomorphism, then the map $h^{-1}: X_{B} \rightarrow X_{A}$ is continuous. It is also easy to check that this correspondence is one to one.

Next we will discuss the duality between join preserving maps between Boolean algebras and special relations on corresponding Stone spaces. Let $X$ and $Y$ be Stone spaces. A relation $R \subseteq X \times Y$ is called point closed if $R[x]=\{y \in Y: x R y\}$ is a closed set for every $x \in X$. We say that $R$ is a clopen relation if for every clopen $U \subseteq Y$ the set $\langle R\rangle U=\{x \in X: R[x] \cap U \neq \emptyset\}$ is a clopen subset of $X$.

Theorem 2.3. (see e.g., [8]) There is a one-to-one correspondence between join preserving maps between Boolean algebras and point-closed and clopen relations on their dual Stone spaces. Moreover, on finite Stone spaces all relations are point-closed and clopen. 
Proof. (Sketch) (1) Let $h: A \rightarrow B$ be a join preserving map, that is, for all $a, b \in A$ we have $h(0)=0$ and $h(a \vee b)=h(a) \vee h(b)$. Let $X_{A}$ and $X_{B}$ be the Stone spaces dual to $A$ and $B$, respectively. We define $R_{h} \subseteq X_{B} \times X_{A}$ by

$$
x R_{h} y \text { iff } y \subseteq h^{-1}(x)
$$

or, equivalently, $x R_{h} y$ iff $(a \in y$ implies $h a \in x) .{ }^{1}$ Conversely, if $R \subseteq X_{B} \times X_{A}$ is a point-closed and clopen relation, then $\langle R\rangle$ is the desired map $\operatorname{Clp}\left(X_{A}\right) \rightarrow \operatorname{Clp}\left(X_{B}\right)$.

Vietoris spaces and their duals, defined below, are central to our investigations.

Definition 2.4 (Functor $V$ ). Let $B$ be a Boolean algebra. Let $V(B)$ be the free Boolean algebra over the set $\{\triangleright a: a \in B\}$ modulo the equations, for all $a, b \in B$,
(1) $\diamond 0=0$
(2) $\diamond(a \vee b)=\diamond a \vee \diamond b$

Thus, $V$ is a functor on Boolean algebras. Now we define the dual to $V$ on Stone spaces.

Definition 2.5 (Functor $K)$ ). For every Stone space $X$ we let $K(X)$ be the set of all closed subsets of $X$ equipped with a topology a subbasis of which is given by the sets

$$
\square(U)=\{F \in K(X): F \subseteq U\} \quad \bigotimes(U)=\{F \in K(X): F \cap U \neq \emptyset\}
$$

where $U$ ranges over clopen subsets of $X$.

The next theorem shows that the two definitions are dual to each other.

Theorem 2.6. [19, Proposition 4.6] Let $B$ a Boolean algebra and $X$ its dual Stone space. Then the algebra $V(B)$ is dual to $K(X)$. If $B$ is finite, $V(B)$ is dual to $\mathcal{P}(X)$.

It follows from the definition of $V(B)$ that a map $\diamond: B \rightarrow V(B)$ mapping each element $a \in B$ to $\diamond a$ is join-preserving. The next proposition characterises the relation on $X \times K(X)$ which is dual to $\diamond$. We just need to observe that $R_{\diamond}$ defined as in the proof of Theorem 2.3 is $\in$.

Proposition 2.7. Let $R_{\diamond} \subseteq K\left(X_{A}\right) \times X_{A}$ be the relation corresponding to the joinpreserving map $\diamond: B \rightarrow V(B)$. Then for every $U \in K(X)$ and $x \in X_{A}$ we have $U R_{\diamond} x$ iff $x \in U$.

\subsection{Priestley duality for distributive lattices}

We briefly review the duality between distributive lattices and Priestley spaces (Stone spaces with special partial orders). Recall that a subset $U$ of an ordered set $(X, R)$ is called an upset if for every $x, y \in X$ we have $x \in U$ and $x R y$ imply $y \in U$. The complement of an upset is called a downset. A relation $R$ on a Stone space $X$ is said to satisfy the Priestley separation axiom if

$\neg(x R y)$ implies there exists a clopen upset $U$ such that $x \in U$ and $y \notin U$.

\footnotetext{
${ }^{1}$ Reading $c \in z$ as $z$ satisfies $c$, we see that $h$ acts here as a modal $\diamond$.
} 
Definition 2.8. A pair $\mathbb{X}=(X, R)$ is called a Priestley space if $X$ is a Stone space and $R$ a partial order satisfying the Priestley separation axiom.

For every Priestley space $\mathbb{X}=(X, R)$ we let $\operatorname{ClpUp}(\mathbb{X})$ denote the set of all clopen upsets of $\mathbb{X}$. We also denote by $U p(\mathbb{X})$ the set of all upsets of $\mathbb{X}$.

Theorem 2.9. (see, e.g, $[14,11.23]$ ) For every distributive lattice $D$ there is a Priestley space $\mathbb{X}_{D}$ such that $D$ is isomorphic to $\left(C \operatorname{lpUp}\left(\mathbb{X}_{D}\right), \cup, \cap, \emptyset\right)$. If $D$ is finite, then $\mathbb{X}_{D}$ is finite and $\operatorname{ClpUp}\left(\mathbb{X}_{D}\right)=\mathrm{Up}\left(\mathbb{X}_{D}\right)$.

Let DL be the category of distributive lattices and lattice homomorphisms. Let also Priest denote the category of Priestley spaces and continuous order-preserving maps. We have the following analogue of Theorem 2.2.

Theorem 2.10. (see, e.g, $[14,11.30]$ ) $\mathrm{DL} \simeq$ Priest $^{o p}$.

Next we will briefly discuss the connection of meet and join preserving maps with Priestley relations. For a relation $R \subseteq X \times Y$ and $U \subseteq Y$ we let $[R] U=\{x \in X$ : $R[x] \subseteq U\}$. Let $\mathbb{X}=(X, R)$ and $\mathbb{Y}=(Y, S)$ be Priestley spaces. A relation $Q \subseteq X \times Y$ is called clopen increasing (resp. clopen decreasing) if for every $x \in X$ the set $Q[x]$ is a closed upset of $\mathbb{Y}$ (resp. a closed downset of $\mathbb{Y}$ ) and for every clopen upset $U$ of $\mathbb{Y}$ the set $[Q] U$ is a clopen upset of $\mathbb{X}$ (resp. $\langle Q\rangle U$ is a clopen downset of $\mathbb{X}$ ).

Theorem 2.11. (eg [11]) There is a one-to-one correspondence between join preserving (resp. meet preserving) maps between distributive lattices and clopen increasing (resp. clopen decreasing) relations on their dual Priestley spaces. Moreover, on finite Priestley spaces a relation $Q$ is clopen increasing (clopen decreasing) iff $Q[x]$ is an upset (resp. a downset) and $[Q]($ resp. $\langle Q\rangle)$ maps upsets to upsets.

\section{Vietoris construction for Priestley spaces and distributive lattices}

Definition 2.12. For every distributive lattice $D$ let $V(D)$ denote the free distributive lattice over the set $\{\diamond a: a \in D\} \cup\{\square a: a \in D\}$ modulo the equations

$$
\begin{array}{ll}
\text { 1. } \diamond 0=0, & \square 1=1, \\
\text { 2. } \diamond(a \vee b)=\diamond a \vee \diamond b, & \square(a \wedge b)=\square a \wedge \square b, \\
\text { 3. } \square(a \vee b) \leq \square a \vee \diamond b, & \square a \wedge \diamond b \leq \diamond(a \wedge b) .
\end{array}
$$

Next we describe the dual construction of the Vietoris space for Priestley spaces [27]. Let $\mathbb{X}=(X, R)$ be a Priestley space. A set $F \subseteq X$ is called convex if for every $x, y, z \in X$ if $x, y \in F$ and $x R z$ and $z R y$, then $z \in F$. For every Priestley space $\mathbb{X}=(X, R)$ let $\operatorname{Conv}(\mathbb{X})$ denote the set of all closed convex subsets of $\mathbb{X}$. We define a topology on $\operatorname{Conv}(\mathbb{X})$ a basis of which is given by the Boolean closure of the sets

$$
\mathbb{\square}(U)=\{F \in \operatorname{Conv}(\mathbb{X}): F \subseteq U\} \quad \nabla(U)=\{F \in \operatorname{Conv}(\mathbb{X}): F \cap U \neq \emptyset\}
$$

where $U$ ranges over clopen upsets of $\mathbb{X}^{2}{ }^{2}$ Moreover, for every $Y, Z \in \operatorname{Conv}(\mathbb{X})$ we define the so-called Egli-Milner order $R^{E M}$ by

\footnotetext{
${ }^{2}$ We note that this definition of topology on the set of closed and convex subsets of a Priestley space together with Theorem 2.13 below solves the problem raised in [27, Section 7.1] on how to define an analogue of the Vietoris topology on the set of closed and convex subsets of a Priestley space.
} 


$$
Y R^{E M} Z \text { iff } Y \subseteq\langle R\rangle Z \text { and } Z \subseteq\langle\check{R}\rangle Y .
$$

where $\check{R}$ is the converse of $R$. Then $\left(\operatorname{Conv}(\mathbb{X}), R^{E M}\right)$ is a Priestley space. The next theorem, which is the Priestley space version of a theorem of Johnstone [20] (see also Palmigiano [27]), shows that the convex set construction on Priestley spaces is the dual to $V$.

Theorem 2.13. Let $D$ be a distributive lattice and $\mathbb{X}=(X, R)$ be its dual Priestley space. Then $\left(\operatorname{Conv}(\mathbb{X}), R^{E M}\right)$ is the Priestley space dual to $V(D)$.

As in the case of modal algebras, we have join-preserving and meet-preserving maps $\diamond$ and $\square$ from $D$ to $V(D)$, mapping every element $a \in D$ to $\diamond a$ and $\square a$, respectively.

Proposition 2.14. Let $R_{\diamond}, R_{\square} \subseteq \operatorname{Conv}\left(\mathbb{X}_{\mathbb{A}}\right) \times \mathbb{X}_{\mathbb{A}}$ be the relations corresponding to $\diamond: D \rightarrow V(D)$ and $\square: D \rightarrow V(D)$, respectively. Then $R_{\diamond}=R_{\square}$ and for every $U \in \operatorname{Conv}(\mathbb{X})$ and $x \in X$ we have $U R_{\diamond} x$ iff $x \in U$.

\section{Modal algebras and distributive modal algebras}

In this section we recall the definitions of modal and distributive modal algebras. We also look at the dual order-topological spaces of these algebras.

Modal algebras A modal algebra (see e.g.[8, 5.2]) is a pair $\left(B, \diamond_{B}\right)$ such that $B$ is a Boolean algebra and $\diamond_{B}: B \rightarrow B$ is a unary operator called a modal operator satisfying the equations of Definition 2.4. We also use a shorthand $\square_{B} a=-\diamond_{B}-a$, for every $a \in B$. Next we recall the representation theorem for modal algebras.

Definition 3.1. (see e.g., [8, Definition 5.65 and Proposition 5.83]) A pair $(X, R)$ is called a modal space if $X$ is a Stone space and $R \subseteq X \times X$ is a point-closed and clopen relation. ${ }^{3}$

Therefore, for every modal space, the algebra $(\operatorname{Clp}(X),\langle R\rangle)$ is a modal algebra. Moreover, every modal algebra can be represented in this way.

Theorem 3.2. (see, e.g., [8, Theorem 5.43]) For every modal algebra $\left(B, \diamond_{B}\right)$ there exists a modal space $(X, R)$ such that $\left(B, \diamond_{B}\right)$ is isomorphic to $(\mathrm{Clp}(X),\langle R\rangle)$.

The modal space $(X, R)$ is called the dual of $\left(B, \diamond_{B}\right)$. Modal spaces can also be seen as coalgebras for the Vietoris functor $K$. In particular, every modal space can be represented as a Stone space $X$ together with a continuous map $R: X \rightarrow K(X)$. The fact that $R$ is well defined corresponds to $R[x]$ being closed, and $R$ being continuous is equivalent to $R$ being a clopen relation. For the details we refer to [22].

Distributive modal algebras Lacking complements, one needs to represent both $\diamond$ and $\square$. A distributive modal algebra (see e.g., [11]) is a triple $\left(D, \diamond_{D}, \square_{D}\right)$ such that $\diamond_{D}: D \rightarrow D$ and $\square_{D}: D \rightarrow D$ are unary operations satisfying for every $a, b \in D$ the equations of Definition 2.12 .

\footnotetext{
${ }^{3}$ Some authors also call modal spaces descriptive frames; see e.g., [8].
} 
Definition 3.3. A triple $(X, R, Q)$ is called a modal Priestley space if $\mathbb{X}=(X, R)$ is a Priestley space and $Q \subseteq X \times X$ is a relation such that

1. $Q[x]$ is closed and convex for every $x \in X$; i.e., $Q[x] \in \operatorname{Conv}(\mathbb{X})$.

2. $\langle Q\rangle U \in \operatorname{ClpUp}(\mathbb{X})$ and $[Q] U \in \operatorname{ClpUp}(\mathbb{X})$ for every $U \in \operatorname{ClpUp}(\mathbb{X})$.

Theorem 3.4. (see [11]) For every distributive modal algebra $(D, \diamond, \square)$ there exists a modal Priestley space $(X, R, Q)$ such that $(D, \diamond, \square)$ is isomorphic to $(\operatorname{ClpUp}(X)$, $\langle R\rangle,[R])$.

We mention here that the modal Priestley spaces can be seen as coalgebras for a functor Conv on Priestley spaces. In particular, every modal Priestley space can be represented as a Priestley space $\mathbb{X}$ together with an order-preserving and continuous map $Q: \mathbb{X} \rightarrow$ $\operatorname{Conv}(\mathbb{X})$. That $Q[x]$ is closed and convex guarantees that $Q$ is well defined. $Q$ being order-preserving and continuous is equivalent to $\langle R\rangle$ and $[R]$ being well-defined maps on $\operatorname{ClpUp}(\mathbb{X})$. For the details we refer to [27].

We close this section by mentioning the connection between modal algebras and modal logic: a (positive) modal formula $\varphi$ is a theorem of the basic modal logic $\mathbf{K}$ iff $\varphi$ is valid in every (distributive) modal algebra.

\section{Main construction}

As observed by Abramsky [2] and Ghilardi [18] the category of modal algebras is isomorphic to the category $\operatorname{Alg}(V)$ of algebras for the functor $V$ and, therefore, the free modal algebras can be obtained by a standard construction in category theory, the initial algebra sequence. Indeed, under fairly general circumstances [4], for a functor $L$ on a category $\mathcal{C}$, the $L$-algebra $L_{\omega}$ free over $C \in \mathcal{C}$ is the colimit of the sequence $\left(L_{n}\right)_{n<\omega}$

$$
L_{0} \stackrel{e_{0}}{\longrightarrow} L_{1} \stackrel{e_{1}}{\longrightarrow} L_{2} \quad \cdots \quad L_{\omega}
$$

where $L_{0}=0$ is the initial object of $\mathcal{C}$ and $L_{n+1}=(C+L)\left(L_{n}\right)$ and $e_{n+1}=(C+$ $L)\left(e_{n}\right)$. Due to $L_{\omega}$ being a colimit, there is a canonical morphism $(C+L)\left(L_{\omega}\right) \rightarrow L_{\omega}$, the components of which provide the insertion of generators $C \rightarrow L_{\omega}$ and the $L$-algebra structure $L\left(L_{\omega}\right) \rightarrow L$. The same result can also be obtained from a slightly different sequence, the one used by [18], which is more convenient for our purposes

$$
L_{0}=C, L_{n+1}=(C+L)\left(L_{n}\right), \quad e_{0}: C \rightarrow C+L(C), e_{n+1}=(C+L)\left(e_{n}\right)
$$

In this paper we are interested in the case where $\mathcal{C}$ is a variety ${ }^{4} \mathbf{V}$ and $L$ encodes a signature that extends the signature $\Sigma_{\mathbf{V}}$ of $\mathbf{V}$ by additional operations $\Sigma^{\prime}$ and the equations $E_{\mathbf{V}}$ of $\mathbf{V}$ by additional equations $E^{\prime}$. The terms in $E^{\prime}$ may use the operations built from the combined signature $\Sigma+\Sigma^{\prime}$. We say that an equation in $E^{\prime}$ is of rank 1 if every variable is under the scope of exactly one occurrence of an operation in $\Sigma^{\prime}$. For example, $\diamond p \rightarrow \square p$ is of rank 1 , but $p \rightarrow \diamond p$ and $\diamond \diamond p \rightarrow \diamond p$ are not. The precise

\footnotetext{
${ }^{4}$ For us, a variety is given by operations of finite arity and equations.
} 
relationship between $L$-algebras and algebras for an extended signature is studied in [23]. Roughly speaking, there is a one-to-one correspondence between functors $L$ : $\mathbf{V} \rightarrow \mathbf{V}$ and extensions of $\mathbf{V}$ by operations $\Sigma^{\prime}$ and equations of rank $1 E^{\prime}$; under this correspondence, $\operatorname{Alg}(L)$ is isomorphic to the variety defined by operations $\Sigma_{\mathbf{V}}+\Sigma^{\prime}$ and equations $E_{\mathbf{V}}+E^{\prime}$. In other words, a variety is isomorphic to $\operatorname{Alg}(L)$ iff it is axiomatized by equations of rank 1 .

The basic construction we will describe is a variation of the sequence (2) which is both more special and more general. More special, because we take $\mathcal{C}$ to be a variety $\mathrm{V}$, more general because we consider sequences whose step-wise construction is not necessarily given by a functor as in (2), ie, for the time being, additional equations not of rank 1 are allowed.

Let $\mathbf{V}$ be a variety of algebras, let $\mathbf{V}_{f}$ be a variety obtained from $\mathbf{V}$ by expanding the signature of $\mathbf{V}$ by an operator $f$ and let $\mathrm{Ax}$ be a set of axioms involving terms built from the operations of $\mathbf{V}$ and $f$. In other words, the algebras in $\mathbf{V}_{f}$ are the pairs $(A, f)$, where $A \in \mathbf{V}$ and $f: A \rightarrow A$ is a map satisfying the axioms in Ax. Further, we let $E q(\mathbf{V})$ and $E q\left(\mathbf{V}_{f}\right)$ be the equational theories of $\mathbf{V}$ and $\mathbf{V}_{f}$, respectively. For every $n \in \omega$ we will construct the $n$-generated free $\mathbf{V}_{f}$-algebra as a colimit of $n$-generated $\mathbf{V}$-algebras $A_{0}-i_{0} \rightarrow A_{1}-i_{1} \rightarrow \cdots . A_{0}$ is the $n$-generated free $\mathbf{V}$-algebra, and each $A_{k+1}$ is obtained from $A_{k}$ by freely adjoining to it the operator $f$. In other words, for each $k \in \omega$ the algebra $A_{k}$ will be the algebra of all the non- $E q\left(\mathbf{V}_{f}\right)$-equivalent terms of degree $\leq k$. Moreover, for each $k \in \omega$, there are two maps $i_{k}$ and $f_{k}$ between $A_{k}$ and $A_{k+1}$. Since $A_{k}$ is the algebra of all terms of degree $\leq k$ and $A_{k+1}$ is the algebra of all terms of degree $\leq k+1$, there is an embedding of $A_{k}$ into $A_{k+1}$. The map $i_{k}$ will be this embedding. Each term of degree $m$, for $m \leq k$ can be turned into a term of degree $m+1 \leq k+1$, by adjoining to it the operator $f$. The map $f_{k}$ is exactly the map that adjoins $f$ to each element of $A_{k}$. The operator $f_{\omega}: A_{\omega} \rightarrow A_{\omega}$ is obtained by lifting the maps $f_{k}: A_{k} \rightarrow A_{k+1}$ to $A_{\omega}$.

The technical details are as follows. We fix a set $\mathrm{P}=\left\{p_{1}, \ldots, p_{n}\right\}$ of variables (or atomic propositions) in the language of $\mathbf{V}$. All the terms that we consider are build from $\mathrm{P}$ using the operations of $\mathbf{V}$ and $f$. For each $k \in \omega$, let $S_{k}$ be the set of all terms in the language of $\mathbf{V}_{f}$ of degree $\leq k$, that is, of all terms that do not contain nestings of ' $f$ ' deeper than $k$. We say that an equation $s=t$, where $s$ and $t$ are terms, is deduced in $\mathbf{V}$ (resp. $\mathbf{V}_{f}$ ) from $\Gamma$ and write $\Gamma \vdash_{\mathbf{V}} s=t$ (resp. $\Gamma \vdash_{\mathbf{V}_{f}} s=t$ ) if $s=t$ is deduced from $\Gamma$ in the equational theory of $\mathbf{V}$ (resp. $\mathbf{V}_{f}$ ). Let $\equiv \mathbf{V}_{f}$ be the relation on $S_{k}$ defined by $s \equiv \mathbf{V}_{f} t$ iff $\vdash \mathbf{V}_{f} s=t$. Using the notation above we make the following

Definition 4.1. The sequence $\left(T_{k}\right)_{k<\omega}$ is the sequence $\left(L_{k}\right)_{k<\omega}$, see (2), where $C$ is the free $\mathbf{V}$-algebra over $P$ and $L: \mathbf{V} \rightarrow \mathbf{V}$ maps $A$ to the free algebra over $\{$ fa $\mid a \in A\}$. The sequence $\left(A_{k}\right)_{k<\omega}$ is the quotient of $\left(T_{k}\right)_{k<\omega}$ by $\equiv_{\mathbf{V}_{f}}$.

The algebra $A_{k}$ is the algebra of (equivalence classes of) terms of degree $\leq k, A_{k+1}$ is the algebra of terms of degree $\leq k+1$ and the $i_{k}: A_{k} \rightarrow A_{k+1}$ obtained from quotienting the $e_{k}$ of (2) are the obvious embeddings. Moreover, we define $f_{k}: A_{k} \rightarrow A_{k+1}$

\footnotetext{
${ }^{5}$ It is straightforward to replace $f$ by a set of operations of finite arity. Here we consider only one unary operator to keep notation simple.
} 
to be the quotients of the maps $T_{k} \rightarrow L\left(T_{k}\right) \rightarrow T_{0}+L\left(T_{k}\right)$ (insertion of generators followed by injection into a coproduct). Because of $i_{k+1} \circ f_{k}=f_{k+1} \circ i_{k}$, the $f_{k}$ give rise, in the underlying category of sets, to a cocone over $\left(A_{k}\right)_{k<\omega}$, equipping $A_{\omega}$ with a $\mathbf{V}_{f}$-algebra structure $f_{\omega}: A_{\omega} \rightarrow A_{\omega}$. More concretely, $f_{k}$ maps a term $t$ of degree $k$ to the term $f(t)$ of degree $k+1$; and, since each $a \in A_{\omega}$ comes from some $A_{k}$, we can write $f_{\omega}(a)=f_{k}(a)$ for some $k$.

Theorem 4.2. The colimit of $\left(A_{k}\right)_{k<\omega}$ is the free $n$-generated $\mathbf{V}_{f}$-algebra.

Note that if $\mathbf{V}$ is locally finite (ie the finitely generated algebras are finite), then each $A_{k}$ is finite. Thus, if $\mathbf{V}$ is locally finite we can approximate every finitely generated free $\mathbf{V}_{f}$ algebra by finite $n$-generated $\mathbf{V}$ algebras. This is the case in our examples, where $\mathbf{V}$ is either the variety $\mathbf{B A}$ of Boolean algebras or the variety $\mathbf{D L}$ of distributive lattices.

The role of rank 1 axioms The equational reasoning needed to determine whether two terms are identified in $A_{k}$ may involve terms of degree larger than $k$. We therefore define $s \equiv_{\mathrm{Ax}}^{k} t$ if, in the equational logic for the signature of $\mathbf{V}_{f}$, the equation $s=t$ has a proof from the axioms Ax that only uses terms of degree $\leq k$.

Definition 4.3. The sequence $\left(A_{k}^{\prime}\right)_{k<\omega}$ is the quotient of $\left(T_{k}\right)_{k<\omega}$ by $\left(\equiv_{\mathrm{Ax}}^{k}\right)_{k<\omega}$.

Theorem 4.4. The colimit of $\left(A_{k}^{\prime}\right)_{k<\omega}$ is the free n-generated $\mathbf{V}_{f}$-algebra.

Note that the $A_{k}$ are determined by the equational theory $E q\left(\mathbf{V}_{f}\right)$ whereas the $\left(A_{k}^{\prime}\right)$ depend on the particular axiomatisation Ax. Moreover, in contrast to the $i_{k}: A_{k} \rightarrow A_{k+1}$, the $i_{k}^{\prime}: A_{k}^{\prime} \rightarrow A_{k+1}^{\prime}$ need not be injective. But if they are, one often can deduce desirable properties like decidability, normal forms, and others as shown by Ghilardi [18]. The following gives a sufficient condition. For a detailed definition of $L$ below see [21, Section 4.1.3].

Theorem 4.5. Let $L$ be the functor on $\mathbf{V}$ where $L(A)$ is the free $\mathbf{V}$-algebra generated by $\{f a \mid a \in A\}$ modulo the axioms $\mathrm{Ax}$. If $\mathrm{Ax}$ is of rank 1, the sequences $\left(A_{k}\right)_{k<\omega}$, $\left(A_{k}^{\prime}\right)_{k<\omega}$ and $\left(L_{k}\right)_{k<\omega}($ see $(2))$ coincide.

In particular, we will exploit that for rank 1 axioms, the morphisms $i_{k}^{\prime}: A_{k}^{\prime} \rightarrow A_{k+1}^{\prime}$ are injective.

\section{Free modal and distributive modal algebras}

We now combine Sections 2 - 4. For modal (distributive) algebras, the axioms Ax of Section 4 are of rank 1 (see Definitions 2.4 and 2.12) and Theorem 4.5 applies.

Free modal algebras Let $B_{0}$ be the $n$-generated free Boolean algebra, that is, $B_{0}$ is isomorphic to the powerset of a $2^{n}$-element set (eg [19, 4.9]). Let $X_{0}$ be the dual of $B_{0}$. According to the construction discussed in the previous section we let $L=B_{0}+V$ in (1), that is,

$$
B_{k+1}=B_{0}+V\left(B_{k}\right) \text {. }
$$

The maps $i_{k}, \diamond_{k}: B_{k} \rightarrow B_{k+1}$ are as in Section 4. From Theorem 4.2 we obtain 
Corollary 5.1. The algebra $\left(B_{\omega}, \diamond_{\omega}\right)$ obtained from the colimit of $\left(B_{k}\right)_{k<\omega}$ is the free modal algebra over $B_{0}$.

Now we will look at the dual of $\left(B_{\omega}, \diamond_{\omega}\right)$. Let $X_{0}$ be a $2^{n}$ element set (the dual of $\left.B_{0}\right)$ and (because of the duality of $\mathcal{P}$ and $V$ (Theorem 2.6) and of $\times$ and + )

$$
X_{k+1}=X_{0} \times \mathcal{P}\left(X_{k}\right) .
$$

Theorem 5.2. The sequence $\left(X_{k}\right)_{k<\omega}$ with maps $\pi_{k}: X_{0} \times \mathcal{P}\left(X_{k}\right) \rightarrow X_{k}$ defined by

$$
\pi_{k}(x, A)=\left(x, \pi_{k-1}[A]\right)
$$

is dual to the sequence $\left(B_{k}\right)_{k<\omega}$ with maps $i_{k}: B_{k} \rightarrow B_{k+1}$. In particular, the $\pi_{k}$ are surjective. Moreover, the relation $R_{k} \subseteq\left(X_{0} \times \mathcal{P}\left(X_{k}\right)\right) \times X_{k}$ defined by

$$
(x, A) R_{k} y \text { iff } y \in A
$$

is dual to $\diamond_{k}: B_{k} \rightarrow B_{k+1}$ (see Theorem 2.3).

Remark 5.3. An element $x=(l, S) \in X_{k+1}$ can be understood as a tree with the root labelled by an element $l \in X_{0}$ and the children being the elements of $S \in \mathcal{P}\left(X_{k}\right)$. These trees have a rich history and have been studied, for example, by $[3,2,6,18,5$, $31]$.

Corollary 5.4. The modal space $\left(X_{\omega}, R_{\omega}\right)$, where $X_{\omega}$ is the limit in Stone of the family $\left\{X_{k}\right\}_{k \in \omega}$ with the maps $\pi_{k+1}: X_{k+1} \rightarrow X_{k}$, and $R_{\omega}$ is defined by $\left(x_{i}\right)_{i \in \omega} R_{\omega}\left(y_{i}\right)_{i \in \omega}$ if $x_{k+1} R_{k} y_{k}$ for each $k \in \omega$ is (isomorphic to) to the dual of $\left(B_{\omega}, \diamond_{\omega}\right)$.

Remark 5.5. Note that $\left(X_{\omega}, R_{\omega}\right)$ is isomorphic to the canonical model of the basic modal logic $\mathbf{K}$; see [8, Section 5]. Therefore, a formula of modal logic is a theorem of $\mathbf{K}$ iff it is satisfiable in $\left(X_{\omega}, R_{\omega}\right)$. Moreover, $\left(X_{\omega}, R_{\omega}\right)$ is also the $K$-coalgebra cofree over $X_{0}$.

Free distributive modal algebras Let $D_{0}$ be the free $n$-generated distributive lattice and $\mathbb{X}_{0}$ be its dual poset, that is, $\mathbb{X}=(\mathcal{P}(\mathbf{n}), \subseteq)$, where $\mathbf{n}=\{0, \ldots, n-1\}$ is an $n$-element set (eg $[19,4.8])$. According to the construction discussed in the previous section we let $D_{k+1}=D_{0}+V\left(D_{k}\right)$. where $V$ is the Vietoris functor for distributive lattices. The maps $i_{k}, \diamond_{k}: D_{k} \rightarrow D_{k+1}$ are as in Section 4. From Theorem 4.2 we obtain

Corollary 5.6. The algebra $\left(D_{\omega}, \diamond_{\omega}\right)$ obtained from the colimit of $\left(D_{k}\right)_{k<\omega}$ is the free modal distributive algebra over $D_{0}$.

For the dual of $\left(D_{\omega}, \searrow_{\omega}\right)$, Theorem 2.13 leads us to define $\mathbb{X}_{k+1}=\mathbb{X}_{0} \times \operatorname{Conv}\left(\mathbb{X}_{k}\right)$.

Theorem 5.7. The sequence $\left(\mathbb{X}_{k}\right)_{k<\omega}$ with $\pi_{k}: \mathbb{X}_{0} \times \operatorname{Conv}\left(\mathbb{X}_{k}\right) \rightarrow \mathbb{X}_{k}$ defined by $\pi_{k}(x, A)=\left(x, \pi_{k-1}[A]\right)$ is dual to the sequence $\left(D_{k}\right)_{k<\omega}$ with maps $i_{k}: D_{k} \rightarrow D_{k+1}$. In particular, the $\pi_{k}$ are surjective. Moreover, the relation $Q_{k} \subseteq\left(\mathbb{X}_{0} \times \operatorname{Conv}\left(\mathbb{X}_{k}\right)\right) \times \mathbb{X}_{k}$. defined by $(x, U) Q_{k} y$ iff $y \in U$ is dual to $\diamond_{k}: D_{k} \rightarrow D_{k+1}$ (see Theorem 2.11). 
Corollary 5.8. The modal Priestley space $\left(\mathbb{X}_{\omega}, Q_{\omega}\right)$, where $\mathbb{X}_{\omega}$ is the inverse limit in Priest of the family $\left\{\mathbb{X}_{k}\right\}_{k \in \omega}$ with the maps $\pi_{k+1}: \mathbb{X}_{k+1} \rightarrow \mathbb{X}_{k}$ and $Q_{\omega}$ is defined by $\left(x_{i}\right)_{i \in \omega} Q_{\omega}\left(y_{i}\right)_{i \in \omega}$ if $x_{k+1} Q_{k} y_{k}$ for each $k \in \omega$ is (isomorphic to) to the dual of $\left(D_{\omega}, \diamond_{\omega}\right)$.

Similar to the modal case the space $\left(\mathbb{X}_{\omega}, Q_{\omega}\right)$ is isomorphic to the canonical model of the basic positive modal logic and it is the final coalgebra for the functor $\mathbb{X}_{0} \times$ Conv on Priestley spaces.

\section{Applications}

Our first three applications are based on approximating the free algebras (and their duals) by the initial sequence of an appropriate functor as in (2). The last section, indicates how to go beyond rank 1 in a systematic way using Sahlqvist theory, but the details have to be left for future work.

\subsection{Normal forms}

In this section we discuss normal forms for the elements of finitely generated free modal and distributive modal algebras. In logical terms this is equivalent to a normal form representation for the formulas of the corresponding language.

Definition 6.1. Let $\mathbf{V}$ be a variety and $A(n)$ an n-generated free algebra of $\mathbf{V}$. We say that $\mathbf{V}$ admits a normal form representation if for every $a \in A(n)$ there exists a term $t(a)$, effectively computable from $a$, such that for every $a, b \in A(n)$ we have $\vdash_{\mathbf{V}} a=b$ iff $t(a)=t(b)$.

We write $A t(-)$ for the set of atoms of a Boolean algebra and, for $T \subseteq B_{0}$ and $S \subseteq$ $A t\left(B_{k}\right)$, let

$$
\alpha_{S, T}:=\bigwedge_{p \in T} p \wedge \bigwedge_{p \notin T} \neg p \wedge \bigwedge_{\varphi \in S} \nabla \varphi \wedge \square \bigvee_{\varphi \in S} \varphi
$$

Lemma 6.2. $a \in B_{k+1}$ is an atom iff $a=\alpha_{S, T}$ for some $T \subseteq B_{k}$ and $S \subseteq \operatorname{At}\left(B_{k}\right)$.

Similarly, for sets $T \subseteq D_{0}$ and $S \subseteq J\left(D_{k}\right)$, where $J\left(D_{k}\right)$ is the set of all joinirreducible elements of $D_{k}$ we let

$$
\beta_{S, T}:=\bigwedge_{p \in T} p \wedge \bigwedge_{\varphi \in S} \nabla \varphi \wedge \square \bigvee_{\varphi \in S} \varphi
$$

Lemma 6.3. $a \in D_{k+1}$ is join-irreducible iff $a=\beta_{S, T}$ for some $T \subseteq D_{k}$ and $S \subseteq$ $J\left(D_{k}\right)$.

Corollary 6.4. Basic modal logic and basic positive modal logic admit normal form representations. 
Proof. The result follows from above lemmas and the fact that every formula $\varphi$ in $n$ variables can be seen as an element of the $n$-generated free algebra of the corresponding variety. As we showed above, for every element of the $n$-generated free modal or distributive modal algebra, there exists $k \in \omega$ such that $\varphi$ belongs to $B_{k}$ or $D_{k}$, respectively. Every element of a finite Boolean algebra (resp. finite distributive lattice) is a join of atoms (join-irreducible elements) that are below this element. Therefore, we obtain that $\varphi=\bigvee \alpha_{S, T}\left(\right.$ resp. $\left.\varphi=\bigvee \beta_{S, T}\right)$.

Remark 6.5. The formulas $\alpha_{S, T}$ are the so-called Fine's canonical formulas [16,25]. Abramsky [2] and Ghilardi [18] derive these formulas in a way similar to ours.

\subsection{Free modal algebras as temporal algebras}

In this section we will give another corollary of our representations of free modal and distributive modal algebras. We will show that these algebras are reducts of temporal algebras. In case of modal logics this was first observed by Ghilardi [18].

Definition 6.6. A modal algebra $(B, \diamond)$ is a reduct of a temporal algebra if there exist $\square_{P}: B \rightarrow B$ such that for every $a, b \in B$ we have $\diamond a \leq b$ iff $a \leq \square_{P} b$.

A distributive modal algebra $(D, \square, \diamond)$ is a reduct of a temporal algebra if there exist $\square_{P}, \diamond_{P}: D \rightarrow D$ such that for every $a, b \in B$ we have $\diamond a \leq b$ iff $a \leq \square_{P} b$ and $\diamond_{P} a \leq b$ iff $a \leq \square b$.

Theorem 6.7. Let $\mathbf{V}$ be a variety of modal or distributive modal algebras axiomatised by the formulas of rank 1 . Then every finitely generated free $\mathbf{V}$-algebra is a reduct of a temporal algebra.

Proof. (Sketch) We only look at the modal case. Let $\left(B_{\omega}, \nabla_{\omega}\right)$ be the free V-algebra. Then since each $B_{k}$ is finite, the map $\diamond_{k}: B_{k} \rightarrow B_{k+1}$ has a right adjoint $\square_{P}^{k}$ : $B_{k+1} \rightarrow B_{k}$, for every $k \in \omega$. Therefore, all we need to show is that the maps $\square_{P}^{k}$ can be extended to the whole of $B_{\omega}$. For this it is sufficient to prove that $i_{k-1} \square_{P}^{k-1}=\square_{P}^{k} i_{k}$. This equation holds if and only if for every $x \in X_{k}$, the equation $\pi_{k-1}^{-1} R_{k-1}[x]=$ $R_{k} \pi_{k}^{-1}[x]$ holds. Checking that the latter equation is satisfied is easy and is based on the fact that for every $k \in \omega$ the maps $\pi_{k}$ are surjective. We skip the details.

\subsection{Pelczynski compactification}

In this section we characterise the space $X_{\omega}$. We show that $X_{\omega}$ is homeomorphic to the so-called Pelczynski space.

Definition 6.8. (see [28] and [26]) A space $\mathfrak{P}$ is called the Pelczynski space if $\mathfrak{P}=$ $X_{\text {iso }} \cup X_{\text {lim }}$, where $X_{\text {iso }}$ is a countable set of isolated points of $\mathfrak{P}, X_{\text {lim }}$ is the set of limit points of $\mathfrak{P}$, the space $X_{\text {lim }}$ is homeomorphic to the Cantor space $\mathfrak{C}$ and $\bar{X}_{\text {iso }}=$ $\mathfrak{P}$.

Theorem 6.9. The underlying Stone space $X_{\omega}$ of the canonical model $\left(X_{w}, R_{w}\right)$ is homeomorphic to the Pelczynski space $\mathfrak{P}$. 
Proof. (Sketch) The proof uses a result of Barr [6] that the set $X_{\text {iso }}$ of isolated points of $X_{\omega}$ is dense in $X_{\omega}$. We proceed by observing that the set $X_{i s o}$ is countable and that the set $X_{l i m}$ of the limit of points of $X_{\omega}$ is uncountable and contains no isolated points in the topology induced from $X_{\omega}$. Thus [15, 6.2.A.(c)], $X_{\text {lim }}$ is homeomorphic to the Cantor space $\mathfrak{C}$ and therefore $X_{\omega}$ is homeomorphic to the Pelczynski space $\mathfrak{P}$.

Remark 6.10. In fact it is not a coincidence that the final coalgebra for the Vietoris functor is based on the Pelczynski space. We can prove that for every polynomial functor $T$ on Stone spaces, the final coalgebra for $T$ is finite or is homeomorphic to the Cantor space $\mathfrak{C}$, the Alexandroff compactification of a countable discrete space or to the Pelczynski space $\mathfrak{P}$.

\subsection{Modal logics not axiomatised by rank 1 axioms}

In this section we indicate that our method can be extended to logics that are not axiomatised by axioms of rank 1 . As a simple example we consider the logic $\mathbf{T}$ obtained from the basic modal logic $\mathbf{K}$ by adding to it the reflexivity axiom $p \rightarrow \diamond p$ (see also Ghilardi [18, Section 5]). The Kripke frames for this logic are characterised by their accessibility relation being reflexive. Let $\mathbf{V}_{\mathbf{T}}$ be the variety of modal algebras corresponding to $\mathbf{T}$. Since the reflexivity axiom is not of rank 1 , in order to construct finitely generated free $\mathbf{V}_{\mathbf{T}}$-algebra, we need to take quotients of the algebras $B_{k}$ (Section 5). For every $k \in \omega$ we will quotient $B_{k}$ by the relation $\equiv_{\mathrm{Ax}}^{k}, \mathrm{AX}=\{p \rightarrow \diamond p\}$, as in Definition 4.3. In other words, we define the sequence $\left(B_{k}^{\prime}\right)_{k<\omega}$ by letting $B_{0}^{\prime}=B_{0}$ and $B_{k+1}^{\prime}=B_{0}^{\prime}+V\left(B_{k}^{\prime}\right)$ modulo $i_{k} a \rightarrow \diamond_{k} a$, for $a \in B_{k}^{\prime}$.

In dual terms, for every $k \in \omega$ we select a subset $Y_{k}$ of $X_{k}$ such that for every $U \subseteq Y_{k}$, we have $\pi_{k}^{-1}(U) \subseteq\left\langle R_{k}\right\rangle U$. This is equivalent to $\pi_{k}^{-1}(y) \subseteq\langle R\rangle\{y\}$ for every $y \in Y_{k}$. (The fact that we can move from sets to singletons is not a coincidence, it is a consequence of a more general fact that $\varphi \rightarrow \Delta \varphi$ is a Sahlqvist formula [8, Section 3.6]). The latter condition is equivalent to $\pi_{k}(x, A) \in A$, for every $(x, A) \in Y_{k+1}$ and $k \in \omega$. Therefore, $Y_{0}=X_{0}$ and for every $k \in \omega, Y_{k+1}=\left\{(x, A): x \in Y_{0}, A \subseteq\right.$ $\left.Y_{k}, \pi_{k}(x, A) \in A\right\}$. By induction on $k$ we can also show that the restriction of $\pi_{k}$ to $Y_{k}$ is a surjection for every $k \in \omega$, which means that the quotients of $i_{k}$ 's are embeddings. Let $S_{k}=R_{k}\left\lceil Y_{k}\right.$ and $\xi_{k}=\pi_{k}\left\lceil Y_{k}\right.$, for each $k \in \omega$. We arrive at the following theorem.

Theorem 6.11. The modal space $\left(\mathbb{Y}_{\omega}, S_{\omega}\right)$, where $\mathbb{Y}_{\omega}$ is the inverse limit in Stone of the family $\left\{\mathbb{Y}_{k}\right\}_{k<\omega}$ with the maps $\xi_{k+1}: \mathbb{Y}_{k+1} \rightarrow \mathbb{Y}_{k}$ and $S_{\omega}$ is defined by $\left(x_{i}\right)_{i<\omega} S_{\omega}\left(y_{i}\right)_{i<\omega}$ if $x_{k+1} S_{k} y_{k}$ for each $k<\omega$ is (isomorphic to) to the canonical model for the modal logic $\mathbf{T}$.

This example suggests that a similar technique can be applied to other logics axiomatised by Sahlqvist formulas. Studying these questions in detail is one of the directions of future work.

\section{Conclusions and future work}

In this paper we presented a uniform method for constructing free algebras for algebras with operators axiomatised by equations of rank 1 . We applied this general method to 
construct free modal algebras and free distributive modal algebras. We also recalled normal forms for modal logic and derived normal forms for positive modal logic. We list directions of further research.

One is to apply this construction to other non-classical logics for example intuitionistic logic, many-valued logics etc. More generally, one might be able to obtain results for varieties, in particular for locally-finite ones, that do arise from logic.

In the context of modal logic most of the important systems can not be axiomatised by the formulas of rank 1 . Therefore, for describing free algebras for those systems, we need to adjust this method as indicated in Section 6.4. As adding axioms means to take quotients of the algebras $A_{k}$, it corresponds to taking subsets of the $X_{k}$ on the dual side. To do this in a uniform way, one should look at Sahlqvist formulas.

Another interesting direction for further research is to spell out in detail the connection of this approach with the one of Moss [25]. It seems that Moss' filtration type technique has a direct representation in our construction. For various modal logics Moss constructs canonical models of formulas of finite modal degree. These models can be obtained from the models $X_{k}$ by lifting in an appropriate way relations $R_{k}$ between $X_{k+1}$ and $X_{k}$ to $X_{k+1}$.

The procedure to obtain normal forms should generalise to all logics of rank 1 (as long as the axioms are effectively given). This should be related to recent work of Schröder and Pattinson [29] on the complexity of rank 1 logics. Marx and Mikulás [24] also obtain complexity bounds for bi-modal logics by looking into algebras of terms of degree $\leq k$. Obtaining normal forms for logics that are not axiomatised by formulas of rank 1 is another interesting question.

We showed that the canonical model of the basic modal logic is based on the Pelczynski space. For other logics, however, such a characterisation does not exists. So a natural question is what are the underlying Stone spaces of canonical models of other modal logics. As we saw above the canonical model of the basic modal logic is a final coalgebra for the Vietoris functor. So an interesting question is whether the final coalgebra for every finite-set preserving functor is also based on the Pelczynski space.

All these questions hold also for positive modal logic and their variations considered in domain theory. But moreover, the recent work of Bruun and Gehrke [10], which connects ontologies with free distributive algebras with operators, adds another smack to this investigations: The axioms that [10] consider in their paper are of rank one.

\section{References}

1. S. Abramsky. Domain theory in logical form. Ann. Pure Appl. Logic, 51:1-77, 1991.

2. S. Abramsky. A Cook's tour of the finitary non-well-founded sets. In We Will Show Them: Essays in Honour of Dov Gabbay. College Publications, 2005. Presented at BCTCS 1988.

3. P. Aczel. Non-Well-Founded Sets. CSLI, Stanford, 1988.

4. J. Adámek and V. Trnková. Automata and Algebras in Categories. Kluwer, 1990.

5. A. Baltag. STS: A Structural Theory of Sets. PhD thesis, Indiana University, 1998.

6. M. Barr. Terminal coalgebras in well-founded set theory. Theoret. Comput. Sci., 114:299 315, 1993.

7. N. Bezhanishvili. Lattices of Intermediate and Cylindric Modal Logics. PhD thesis, ILLC, University of Amsterdam, 2006. 
8. P. Blackburn, M. de Rijke, and Y. Venema. Modal Logic. CUP, 2001.

9. M. Bonsangue and A. Kurz. Duality for logics of transition systems. In FoSSaCS'05, LNCS 3441, 2005.

10. H. Bruun and M. Gehrke. Distributive lattice-structured ontologies. 2006. Draft.

11. S. Celani and R. Jansana. Priestley duality, a Sahlqvist theorem and a Goldblatt-Thomason theorem for positive modal logic. J. of the IGPL, 7:683-715, 1999.

12. A. Chagrov and M. Zakharyaschev. Modal Logic. OUP, 1997.

13. B. Davey and M. Goldberg. The free p-algebra generated by a distributive lattice. Algebra Universalis, 11:90-100, 1980.

14. B. Davey and H. Priestley. Introduction to Lattices and Order. CUP, 1990.

15. R. Engelking. General Topology. Heldermann Verlag, 1989.

16. K. Fine. Normal forms in modal logic. Notre Dame J. Formal Logic, 16:229-237, 1975.

17. S. Ghilardi. Free Heyting algebras as bi-Heyting algebras. Math. Rep. Acad. Sci. Canada XVI, 6:240-244, 1992.

18. S. Ghilardi. An algebraic theory of normal forms. Ann. Pure Appl. Logic, 71:189-245, 1995.

19. P. Johnstone. Stone Spaces. CUP, 1982.

20. P. Johnstone. Vietoris locales and localic semilattices. In Continuous lattices and their applications, Lecture Notes in Pure and Appl. Math., pages 155-180. New York, 1985.

21. C. Kupke. Finitary Coalgebraic Logics. PhD thesis, ILLC, Amsterdam, 2006.

22. C. Kupke, A. Kurz, and Y. Venema. Stone coalgebras. Theoret. Comput. Sci., 327:109-134, 2004.

23. A. Kurz and J. Rosický. Strongly complete logics for coalgebras. Submitted, electronically available.

24. M. Marx and S. Mikulás. An elementary construction for a non-elementary procedure. Studia Logica, 72:253-263, 2002.

25. L. Moss. Finite models constructed from canonical formulas. Journal of Philosophical Logic, 2007. To appear.

26. S. Oka. The topological types of hyperspaces of 0-dimensional compacta. Topology and its Applications, 149:227-237, 2005.

27. A. Palmigiano. A coalgebraic view on positive modal logic. Theoret. Comput. Sci., 327:175195, 2004.

28. A. Pelczynski. A remark on spaces $2^{X}$ for zero-dimensional X. Bull. Pol. Acad. Sci., 13:85$89,1965$.

29. L. Schröder and D. Pattinson. PSPACE bounds for rank 1 modal logics. In LICS'06, 2006.

30. Y. Venema. Algebras and coalgebras. In P. Blackburn, J. van Benthem, and F. Wolter, editors, Handbook of Modal Logic, pages 331-426. Elsevier, 2007.

31. J. Worrell. On the final sequence of an finitary set functor. Theoret. Comput. Sci., 338:184199, 2005. 